\title{
LOCALIZATION ANALYSIS OF AN ORTHOTROPIC MULTI-SURFACE PLASTICITY MODEL UNDER UNIAXIAL STRESS
}

\author{
Claudio Pagani*, Milan Jirásek, Martin Horák \\ Czech Technical University in Prague, Faculty of Civil Engineering, Department of Mechanics, Thákurova 7, \\ 16629 Prague 6, Czech Republic \\ * corresponding author: claudio.pagani@fsv.cvut.cz
}

\begin{abstract}
Numerical simulations of masonry structures are often based on continuum macromodelling approaches that need constitutive laws able to phenomenologically reproduce the behavior of the material. To capture the deformation process up to failure, appropriate softening laws are needed to take into account the contraction of the yield stress domain caused by cracking and crushing. It is well known that softening may lead to localization of inelastic strain. This paper focuses on localization analysis of an orthotropic macro-scale model in the framework of multi-surface plasticity, which describes the in-plane behavior of masonry structures. Preliminary results reported in this short paper are limited to uniaxial stress states. Analytical localization conditions are first derived for uniaxial stress states with principal axes aligned with the material axes of orthotropy. Then, localization analysis is extended to an arbitrary angle between the principal stress axes and the axes of orthotropy.
\end{abstract}

KEYWORDS: Multi-surface plasticity, localization, weak discontinuity, masonry.

\section{INTRODUCTION}

Masonry structures are characterized by two different failure modes, the in-plane and out-of-plane mechanisms. Regarding the study of in-plane mechanisms, different strategies could be adopted for numerical simulations of the behavior of masonry structures. Among others, continuum macro-modeling is a frequently used approach, in which masonry structures are treated as homogenized continua and, in general, discretized by the finite element method. This kind of modelling needs the choice of a constitutive model, which could be formulated within the framework of nonlinear elasticity, plasticity, damage mechanics, smeared crack models, or their combinations, and its aim is to phenomenologically reproduce the average behavior of masonry.

This paper is focused on a study of an orthotropic model in the framework of multisurface plasticity, with the yield function described as the composition of two different surfaces for the tensile and the compressive behavior [1, 2]. Both failure surfaces evolve according to hardening/softening laws that may lead to localization of plastic strain. Conditions for the onset of localization depend on many factors, such as the stress state, the destabilizing effects of the non-associated flow rule in plasticity, or, in this particular case, the orthotropy of the elastic stress-strain law.

In this paper, the localization analysis of the presented macro-model is carried out under the condition of uniaxial stress in tension and in compression. Conditions for the onset of localization are derived and preferential directions of the emerging weak discontinuity are evaluated.

\section{MODEL DESCRIPTION}

This paper deals with an advanced model for the in-plane failure mechanisms of masonry structures proposed by Lourenço [1, 2, which takes into account the anisotropic structure of masonry and is formulated in the framework of multisurface plasticity.

The analysis can be reduced to two dimensions when plane-stress conditions are assumed. In the following description, the Cartesian axes $x$ and $y$ are considered aligned with the material axes, which, for regular periodic masonry, are the horizontal and vertical ones (bed and head mortar joints, respectively).

In order to avoid pathological mesh sensitivity of the results to the element size, model parameters that affect the stress-strain diagrams in tension and compression are adjusted by means of the equivalent length $l_{e}$. In the original paper, the crack band size was related to the area of the elements:

$$
l_{e}=\alpha_{h} \sqrt{A_{e}}
$$

where $\alpha_{h}$ is 1 or $\sqrt{2}$ for quadratic and linear elements, respectively. This rule, which has no convincing justification, may induce a large error for elongated elements and even for square elements since the crack band size depends also on the orientation with respect to the element sides. In this paper, the projection method is considered, i.e., the effective crack band size is estimated by projecting the element onto the direction perpendicular to the estimated crack band direction [3.

The model requires in total 16 parameters, which are reported in Table 1, and are divided into groups governing orthotropic elasticity, tensile and compres- 
sive plastic behavior. The meaning of the symbols is described in the following sections.

\subsection{Orthotropic Elasticity}

The linear elastic stress-strain relation under planestress conditions can be written as

$$
\sigma=D_{e} \varepsilon
$$

where stress and strain components are stored in column matrices using the Voigt notation:

$$
\boldsymbol{\sigma}=\left[\begin{array}{c}
\sigma_{x} \\
\sigma_{y} \\
\sigma_{x y}
\end{array}\right], \quad \boldsymbol{\varepsilon}=\left[\begin{array}{c}
\varepsilon_{x} \\
\varepsilon_{y} \\
\gamma_{x y}
\end{array}\right]
$$

The elastic stiffness matrix for an orthotropic material reads

$$
\boldsymbol{D}_{e}=\frac{1}{\zeta}\left[\begin{array}{ccc}
E_{x} & \nu_{y x} E_{x} & 0 \\
\nu_{x y} E_{y} & E_{y} & 0 \\
0 & 0 & \zeta G_{x y}
\end{array}\right]
$$

where $\zeta=1-\nu_{x y} \nu_{y x}$ is an auxiliary parameter.

The elastic behavior is characterized by four parameters: $E_{x}, E_{y}, \nu_{x y}$, and $G_{x y}$. The other in-plane Poisson's ratio is determined from symmetry and is given by $\nu_{y x}=\nu_{x y} E_{y y} / E_{x x}$.

The plane stress condition $\left(\sigma_{z}=0\right)$ leads to the following expression for the out-of-plane normal strain:

$$
\varepsilon_{z}=-\frac{1}{\zeta}\left[\left(\nu_{x z}+\nu_{y z} \nu_{x y}\right) \varepsilon_{x}+\left(\nu_{x z} \nu_{y x}+\nu_{y z}\right) \varepsilon_{y}\right]
$$

Hence, additional two Poisson's ratios $\left(\nu_{x z}\right.$ and $\left.\nu_{y z}\right)$ have to be known if one is interested in computing the out-of-plane strain.

\subsection{Plastic Behavior in tension}

An orthotropic Rankine type yield surface is considered for the tensile behavior, with orthotropic softening controlled by a single scalar quantity. The corresponding yield function is defined as

$$
\begin{gathered}
f_{t}\left(\boldsymbol{\sigma}, \kappa_{t}\right)=\frac{\left(\sigma_{x}-\sigma_{t, x}\left(\kappa_{t}\right)\right)+\left(\sigma_{y}-\sigma_{t, y}\left(\kappa_{t}\right)\right)}{2}+ \\
+\sqrt{\left(\frac{\left(\sigma_{x}-\sigma_{t, x}\left(\kappa_{t}\right)\right)-\left(\sigma_{y}-\sigma_{t, y}\left(\kappa_{t}\right)\right)}{2}\right)^{2}+\alpha \tau_{x y}^{2}}
\end{gathered}
$$

Degradation of the yield stress is governed by a single scalar internal variable $\kappa_{t}$. The softening laws

$$
\sigma_{t, i}\left(\kappa_{t}\right)=f_{t, i} \exp \left(-\frac{l_{e} f_{t, i}}{G_{f t, i}} \kappa_{t}\right), \quad i \in\{x, y\}
$$

describe the dependence of the equivalent tensile yield stresses in directions $x$ and $y$ in the form of exponential softening and can be collected into the column matrix

$$
\boldsymbol{q}_{t}=\left[\begin{array}{c}
\sigma_{t, x}\left(\kappa_{t}\right) \\
\sigma_{t, y}\left(\kappa_{t}\right) \\
0
\end{array}\right]
$$

Parameters $f_{t, i}$ and $G_{f t, i}, i \in\{x, y\}$, are the tensile strength and the fracture energy in tension corresponding to the $i$-axis, respectively. To avoid snap-back at the constitutive level, the value of the tensile strength is reduced if the elements are too large, according to the rule

$$
f_{t, i}=\sqrt{\frac{G_{f t, i} E_{i}}{l_{e}}} \quad \text { if } \quad l_{e}>\frac{G_{f t, i} E_{i}}{f_{t, i}^{2}}
$$

Parameter

$$
\alpha=\frac{f_{t, x} f_{t, y}}{\tau_{u, t}^{2}}
$$

controls the shear stress contribution to tensile failure, where $\tau_{u, t}$ is the shear strength in tension.

The flow rule is non-associated and is derived from the plastic potential

$$
\begin{aligned}
& g_{t}\left(\boldsymbol{\sigma}, \kappa_{t}\right)=\frac{\left(\sigma_{x}-\sigma_{t, x}\left(\kappa_{t}\right)\right)+\left(\sigma_{y}-\sigma_{t, y}\left(\kappa_{t}\right)\right)}{2}+ \\
& +\sqrt{\left(\frac{\left(\sigma_{x}-\sigma_{t, x}\left(\kappa_{t}\right)\right)-\left(\sigma_{y}-\sigma_{t, y}\left(\kappa_{t}\right)\right)}{2}\right)^{2}+\tau_{x y}^{2}}
\end{aligned}
$$

The rate of the softening parameter is considered equal to the rate of the maximum principal plastic strain:

$$
\dot{\kappa}_{t}=\dot{\varepsilon}_{1}^{P}=\frac{\dot{\varepsilon}_{x}^{P}+\dot{\varepsilon}_{y}^{P}}{2}+\frac{1}{2} \sqrt{\left(\dot{\varepsilon}_{x}^{P}-\dot{\varepsilon}_{y}^{P}\right)+\left(\dot{\gamma}_{x y}^{P}\right)^{2}}
$$

It is assumed that the hardening regimes in tension and compression are uncoupled, i.e., each of the surfaces is associated with its own hardening variable that grows only if the surface is active. Under this assumption, the expression of $\dot{\kappa}_{t}$ reduces to

$$
\dot{\kappa}_{t}=\dot{\lambda}_{t}
$$

where $\lambda_{t}$ is the plastic multiplier associated with the tensile yield surface.

In conclusion, there are five model parameters controlling the tensile behavior: two strengths, $f_{t, x}$ and $f_{t, y}$, two fracture energies, $G_{f t, x}$ and $G_{f t, y}$ and the parameter $\alpha$ that governs the coupling of shear with the normal stresses.

\subsection{Plastic Behavior in COMPRESSION}

The adopted failure surface in compression corresponds to a Hill-type yield function,

$$
f_{c}=\frac{\sigma_{x}^{2}}{\sigma_{c, x}^{2}\left(\kappa_{c}\right)}+\frac{\beta \sigma_{x} \sigma_{y}+\gamma \tau_{x y}^{2}}{\sigma_{c, x}\left(\kappa_{c}\right) \sigma_{c, y}\left(\kappa_{c}\right)}+\frac{\sigma_{y}^{2}}{\sigma_{c, y}^{2}\left(\kappa_{c}\right)}-1
$$

where $\beta$ is a parameter that controls the coupling between the normal stresses. Parameter $\gamma$ controls the shear stress contribution to failure and is given by

$$
\gamma=\frac{f_{c, x} f_{c, y}}{\tau_{u, c}^{2}}
$$


where $f_{m, x}$ and $f_{m, y}$ are the values of uniaxial compression strength in directions $x$ and $y$, and $\tau_{u, c}$ is the shear strength in compression.

Hardening and softening are controlled by a strainlike internal variable $\kappa_{c}$, defined by the rate equation

$$
\dot{\kappa}_{c}=\frac{1}{\sqrt{\sigma_{c, x}\left(\kappa_{c}\right) \sigma_{c, y}\left(\kappa_{c}\right)}} \boldsymbol{\sigma}^{T} \dot{\varepsilon}_{p}
$$

which, under the assumption of uncoupled hardening regimes in tension and compression, reduces to

$$
\dot{\kappa}_{c}=\dot{\lambda}_{c}
$$

where $\lambda_{c}$ is the plastic multiplier associated with the compressive yield surface.

The values of current compressive yield stress in directions $x$ and $y, \sigma_{c, x}$ and $\sigma_{c, y}$, are evaluated from $\kappa_{c}$ using the hardening-softening law (Figure 1)

$$
\sigma_{c, i}= \begin{cases}\sigma_{i, i}+\left(\sigma_{p, i}-\sigma_{i, i}\right) \sqrt{\frac{2 \kappa_{c}}{\kappa_{p}}-\frac{\kappa_{c}^{2}}{\kappa_{p}^{2}}} & \kappa_{c} \leq \kappa_{p} \\ \sigma_{p, i}+\left(\sigma_{m, i}-\sigma_{p, i}\right)\left(\frac{\kappa_{c}-\kappa_{p}}{\kappa_{m, i}-\kappa_{p}}\right)^{2} & \kappa_{p}<\kappa_{c} \leq \kappa_{m, i} \\ \sigma_{r, i}+\left(\sigma_{m, i}-\sigma_{r, i}\right) \mathrm{e}^{\frac{2 m\left(\kappa_{c}-\kappa_{m}\right)}{\kappa_{m}-\kappa_{p}}} & \kappa_{m, i}<\kappa_{c}\end{cases}
$$

where parameter

$$
m=\frac{\sigma_{m, i}-\sigma_{p}}{\sigma_{m, i}-\sigma_{r, i}}
$$

corresponds to parabolic hardening followed by parabolic and later exponential softening.

This law contains strain-like parameters $\kappa_{p}$, which represents the value of $\kappa_{c}$ at the peak, i.e., at transition from hardening to softening, and $\kappa_{m, x}$ and $\kappa_{m, y}$, which correspond to the transition from parabolic to exponential softening, and also stress-like parameters $\sigma_{i, x}$ and $\sigma_{i, y}$, which are the initial values of yield stress in directions $x$ and $y, \sigma_{p, x}$ and $\sigma_{p, y}$, which are the peak values of yield stress, $\sigma_{m, x}$ and $\sigma_{m, y}$, which are the values of yield stress at transition from parabolic to exponential softening, and $\sigma_{r, x}$ and $\sigma_{r, y}$, which are the values of residual yield stress.

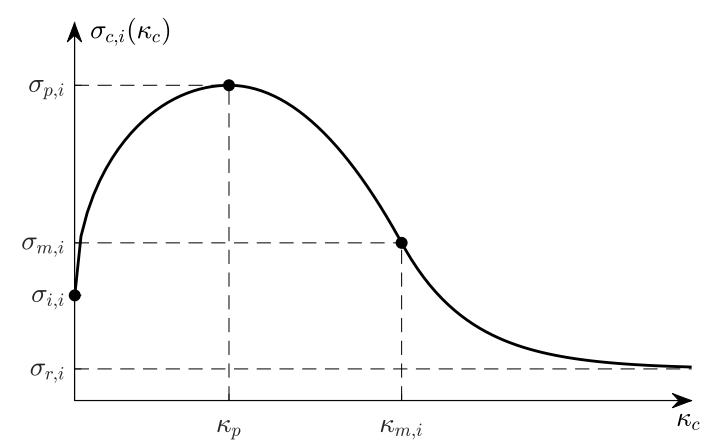

Figure 1. Hardening/softening law for the compressive yield stress
In absence of specific experimental tests, the characteristic yield stress values can be set to

$$
\begin{aligned}
\sigma_{p, i} & =f_{c, i} & \sigma_{i, i} & =\frac{1}{3} f_{c, i} \\
\sigma_{m, i} & =\frac{1}{2} f_{c, i} & \sigma_{r, i} & =\frac{1}{10} f_{c, i}
\end{aligned}
$$

The value of the equivalent plastic strain at peak, $\kappa_{p}$, is considered to be an additional parameter of the model. The value of $\kappa_{m, i}$ depends on the estimated crack band width $l_{e}$ (related to the finite element mesh) and is given by

$$
\kappa_{m, i}=\frac{75}{67} \frac{G_{f c, i}}{l_{e} f_{c, i}}+\kappa_{p}
$$

where $G_{f c, i}$ is the fracture energy in compression corresponding to the $i$-axis. In order to avoid snapback at the constitutive level, the compressive strength is reduced if the elements are too large, according to the formula

$$
f_{c, i}=\sqrt{\frac{75}{67} \frac{G_{f c, i} E_{i}}{l_{e}}} \quad \text { if } \quad \kappa_{m, i}<\frac{f_{c, i}}{E_{i}}+\kappa_{p}
$$

The values of current compressive yield stress in directions $x$ and $y$ could be cast into the column matrix

$$
\boldsymbol{q}_{c}=\left[\begin{array}{c}
\sigma_{c, x}\left(\kappa_{c}\right) \\
\sigma_{c, y}\left(\kappa_{c}\right) \\
0
\end{array}\right]
$$

An associated flow rule for compression regime is used, which means that the rate of plastic strain is given by

$$
\dot{\varepsilon}_{p}=\dot{\lambda}_{c} \frac{\partial f_{c}\left(\boldsymbol{\sigma}, \kappa_{c}\right)}{\partial \boldsymbol{\sigma}}
$$

In summary, six model parameters are used for description of the inelastic behavior in compression: compressive strengths $f_{c, x}$ and $f_{c, y}$, fracture energies $G_{f c, x}$ and $G_{f c, y}$, coupling parameter between normal stresses in two directions, $\beta$, and the equivalent plastic strain value corresponding to the peak, $\kappa_{p}$.

\section{LOCALIZATION ANALYSIS IN PLASTICITY}

Suppose that plastic loading takes place with only one yield surface activated, and let $i$ be the index of the active surface $(i \in\{t, c\})$.

In the following derivation, a comma denotes a partial derivative with respect to the variable following the comma and no sum over repeated indices is considered.

The basic equations characterizing the flow theory of plasticity include the elastic-plastic split,

$$
\varepsilon=\varepsilon_{e}+\varepsilon_{p}
$$

the stress-strain law for the elastic part (2), the yield condition

$$
f_{i}\left(\boldsymbol{\sigma}, \boldsymbol{q}_{i}\right)=0
$$


the flow rule,

$$
\dot{\varepsilon}_{p}=\dot{\lambda}_{i} g_{i, \sigma}
$$

the definition of hardening/softening variable in the rate form,

$$
\dot{\kappa}_{i}=\dot{\lambda}_{i}
$$

the dependence of the yield strength on the hardening variable

$$
\boldsymbol{q}_{i}=\boldsymbol{h}_{i}\left(\kappa_{i}\right)
$$

and the loading/unloading conditions

$$
f_{i} \leq 0, \quad \dot{\lambda}_{i} \geq 0, \quad \dot{\lambda}_{i} f_{i}=0
$$

During plastic flow, the yield function must remain equal to zero, and so the rate of its change is also zero. This consideration leads to the consistency condition,

$$
\dot{\lambda}_{i} \dot{f}_{i}=0
$$

from which the elasto-plastic stiffness matrix can be computed. The resulting formula reads

$$
\begin{aligned}
\boldsymbol{D}_{e p}=\boldsymbol{D}_{e}- & \frac{\boldsymbol{D}_{e}: g_{i, \boldsymbol{\sigma}} \otimes f_{i, \boldsymbol{\sigma}}: \boldsymbol{D}_{e}}{f_{i, \boldsymbol{\sigma}}: \boldsymbol{D}_{e}: g_{i, \boldsymbol{\sigma}}-f_{i, \boldsymbol{q}_{i}} \cdot \boldsymbol{H}_{i}}= \\
& =\boldsymbol{D}_{e}-\frac{1}{h} \boldsymbol{D}_{e}: g_{i, \boldsymbol{\sigma}} \otimes f_{i, \boldsymbol{\sigma}}: \boldsymbol{D}_{e}
\end{aligned}
$$

where

$$
h=f_{i, \boldsymbol{\sigma}}: \boldsymbol{D}_{e}: g_{i, \boldsymbol{\sigma}}-f_{i, \boldsymbol{q}_{i}} \cdot \boldsymbol{H}_{i}
$$

and

$$
\boldsymbol{H}_{i}=\boldsymbol{q}_{i, \kappa_{i}}
$$

The condition of incipient weak discontinuity, as inspired by the early works of Hadamard [4] and Hill [5] and developed, among others, for plasticity 6, 7] and for damage [8, 9], is given by

$$
\left(\boldsymbol{n} \cdot \boldsymbol{D}_{e p} \cdot \boldsymbol{n}\right) \cdot \boldsymbol{m}=\mathbf{0}
$$

where $\boldsymbol{n}$ is the normal to the discontinuity surface, and

$$
\boldsymbol{Q}_{e p}=\boldsymbol{n} \cdot \boldsymbol{D}_{e p} \cdot \boldsymbol{n}
$$

is the elasto-plastic acoustic tensor. According to (36), this tensor is singular at the onset of localization. The eigenvector $\boldsymbol{m}$ corresponding to the zero eigenvalue is called the polarization vector.

The elasto-plastic acoustic tensor can be expressed as

$$
\begin{aligned}
& \boldsymbol{Q}_{e p}=\boldsymbol{n} \cdot \boldsymbol{D}_{e p} \cdot \boldsymbol{n}= \\
& =\boldsymbol{n} \cdot \boldsymbol{D}_{e} \cdot \boldsymbol{n}-\frac{1}{h} \boldsymbol{n} \cdot \boldsymbol{D}_{e}: g_{i, \boldsymbol{\sigma}} \otimes f_{i, \boldsymbol{\sigma}}: \boldsymbol{D}_{e} \cdot \boldsymbol{n}
\end{aligned}
$$

which can be recast in a simpler form

$$
\boldsymbol{Q}_{e p}=\boldsymbol{Q}_{e}-\frac{1}{h} \boldsymbol{b} \otimes \boldsymbol{a}
$$

where

$$
\begin{aligned}
\boldsymbol{Q}_{e} & =\boldsymbol{n} \cdot \boldsymbol{D}_{e} \cdot \boldsymbol{n} \\
\boldsymbol{a} & =\boldsymbol{n} \cdot \boldsymbol{D}_{e}: f_{i, \boldsymbol{\sigma}} \\
\boldsymbol{b} & =\boldsymbol{n} \cdot \boldsymbol{D}_{e}: g_{i, \boldsymbol{\sigma}}
\end{aligned}
$$

After some calculations, by imposing singularity of the elastoplastic acoustic tensor, the condition of an incipient weak discontinuity $(36)$ becomes

$$
-f_{i, \boldsymbol{q}_{i}} \cdot \boldsymbol{H}_{i}=\boldsymbol{a} \cdot \boldsymbol{Q}_{e}^{-1} \cdot \boldsymbol{b}-f_{i, \boldsymbol{\sigma}}: \boldsymbol{D}_{e}: g_{i, \boldsymbol{\sigma}}
$$

Since we are in plane-stress conditions and considering the plane of symmetry perpendicular to the $z$-axis, one can restrict attention to normals $\boldsymbol{n}$ for which

$$
\boldsymbol{n}=\left[\begin{array}{c}
n_{1} \\
n_{2} \\
0
\end{array}\right]
$$

under the normalizing constraint $n_{1}^{2}+n_{2}^{2}=1$. Considering major and minor symmetries of the orthotropic elastic stiffness tensor and the plane-stress condition, the quantities entering in (43) can be simplified.

Namely, the orthotropic linear elastic acoustic tensor is given by

$$
\boldsymbol{Q}_{e}=\left[\begin{array}{cc}
D_{1111} n_{1}^{2}+D_{1212} n_{2}^{2} & \left(D_{1122}+D_{1212}\right) n_{1} n_{2} \\
\left(D_{1122}+D_{1212}\right) n_{1} n_{2} & D_{1212} n_{1}^{2}+D_{2222} n_{2}^{2}
\end{array}\right]
$$

where

$$
\begin{aligned}
D_{1111} & =\frac{E_{x x}}{1-\nu_{x y} \nu_{y x}} \\
D_{2222} & =\frac{E_{y y}}{1-\nu_{x y} \nu_{y x}} \\
D_{1122} & =\frac{\nu_{y x} E_{x x}}{1-\nu_{x y} \nu_{y x}} \\
D_{1212} & =G_{x y}
\end{aligned}
$$

Other important quantities are evaluated as

$$
\begin{aligned}
f_{i, \boldsymbol{\sigma}}: \boldsymbol{D}_{e}: & g_{i, \boldsymbol{\sigma}}=D_{1111} f_{i, \boldsymbol{\sigma}}^{11} g_{i, \boldsymbol{\sigma}}^{11}+ \\
+ & D_{1122}\left(f_{i, \boldsymbol{\sigma}}^{11} g_{i, \boldsymbol{\sigma}}^{22}+f_{i, \boldsymbol{\sigma}}^{22} g_{i, \boldsymbol{\sigma}}^{11}\right)+ \\
& +4 D_{1212} f_{i, \boldsymbol{\sigma}}^{12} g_{i, \boldsymbol{\sigma}}^{12}+D_{2222} f_{i, \boldsymbol{\sigma}}^{22} g_{i, \boldsymbol{\sigma}}^{22}
\end{aligned}
$$

$$
\begin{aligned}
& \boldsymbol{a}=\left[\begin{array}{c}
\left(D_{1111} f_{i, \boldsymbol{\sigma}}^{11}+D_{1122} f_{i, \boldsymbol{\sigma}}^{22}\right) n_{1}+2 D_{1212} f_{i, \boldsymbol{\sigma}}^{12} n_{2} \\
2 D_{1212} f_{i, \boldsymbol{\sigma}}^{12} n_{1}+\left(D_{1122} f_{i, \boldsymbol{\sigma}}^{11}+D_{2222} f_{i, \boldsymbol{\sigma}}^{22}\right) n_{2}
\end{array}\right] \\
& \boldsymbol{b}=\left[\begin{array}{c}
\left(D_{1111} g_{i, \boldsymbol{\sigma}}^{11}+D_{1122} g_{i, \boldsymbol{\sigma}}^{22}\right) n_{1}+2 D_{1212} g_{i, \boldsymbol{\sigma}}^{12} n_{2} \\
2 D_{1212} g_{i, \boldsymbol{\sigma}}^{12} n_{1}+\left(D_{1122} g_{i, \boldsymbol{\sigma}}^{11}+D_{2222} g_{i, \boldsymbol{\sigma}}^{22}\right) n_{2}
\end{array}\right]
\end{aligned}
$$

where $f_{i, \boldsymbol{\sigma}}^{i j}$ and $g_{i, \boldsymbol{\sigma}}^{i j}$ are the $i j$ components of the derivative of the yielding function and the plastic potential, respectively, with respect to stress tensor.

\section{LOCALIZATION ANALYSIS FOR UNIAXIAL STRESS}

Let us consider the case of uniaxial stress (Figure 2 whose direction is inclined by an angle $\phi$ with respect to the $x$-axis that, together with the $y$-axis, forms the pair of orthotropy axes. Let $\theta$ be the angle that the generic normal $\boldsymbol{n}$ to the crack surface forms with the material $x$-axis, so that it can be expressed as

$$
\boldsymbol{n}=\left[\begin{array}{c}
\cos \theta \\
\sin \theta \\
0
\end{array}\right]
$$


The stress tensor matrix in the $O x y$ reference frame corresponding to uniaxial tension of magnitude $\bar{\sigma}$ and rotated by a generic angle $\phi$ with respect to the $x$-axis is given by

$$
\boldsymbol{\sigma}(\bar{\sigma}, \phi)=\boldsymbol{Q} \hat{\boldsymbol{\sigma}} \boldsymbol{Q}^{T}
$$

where

$$
\hat{\boldsymbol{\sigma}}=\left[\begin{array}{cc}
\bar{\sigma} & 0 \\
0 & 0
\end{array}\right] \quad \boldsymbol{Q}=\left[\begin{array}{cc}
\cos \phi & -\sin \phi \\
\sin \phi & \cos \phi
\end{array}\right]
$$

The value $\bar{\sigma}$ should be the one that triggers localization for the given stress angle $\phi$, so it is the value corresponding to the onset of plastic flow and is determined by the condition $f_{i}=0$ where $f_{i}$ is yield function given by (6) or (14) for tension or compression, respectively.

Subsequently, one can substitute the determined value of $\bar{\sigma}$ for a given $\phi$ into the right-hand side of (43) and obtain an expression depending only on the angle $\theta$. The localization angle $\theta_{l o c}$ is determined as the one that maximizes the right-hand side of (43) and the corresponding value is the critical hardening modulus $\left(H_{\text {crit }}=H\left(\theta_{l o c}\right)\right)$. The polarization vector $\boldsymbol{m}$, the eigenvector of the elastoplastic acoustic tensor corresponding to the zero eigenvalue, characterizes the failure mode, ranging from tensile splitting (mode I) with $\boldsymbol{m}=\boldsymbol{n}$ to shear slip (mode II) with $\boldsymbol{m}$ perpendicular to $\boldsymbol{n}$.

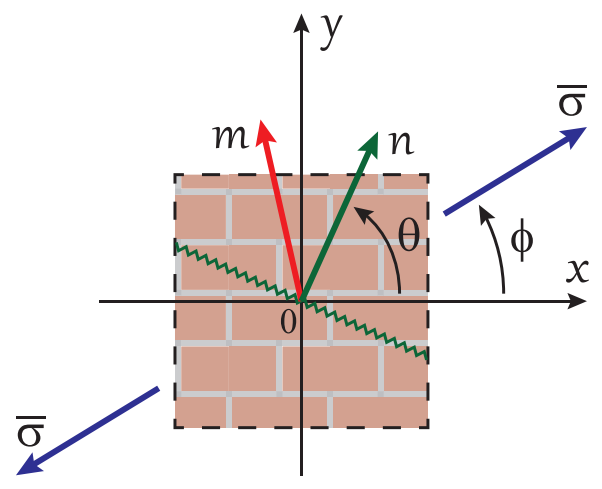

Figure 2. Localization $(n)$ and polarization $(m)$ vectors for uniaxial stress $\bar{\sigma}$ inclined by $\phi$ with respect the material $x$-axis

In the case of uniaxial tension and compression along the $x$ or $y$-axis, the expressions of the critical hardening moduli can be found in a closed form. First, it is useful to define the quantity $\xi$, common to all the expressions:

$$
\begin{aligned}
\xi(\theta)=- & \left(\frac{\sin ^{4} \theta}{E_{x x}}+\frac{\cos ^{4} \theta}{E_{y y}}+\right. \\
& \left.+\frac{E_{x x}-2 G_{x y} \nu_{x y}}{E_{x x} G_{x y}} \sin ^{2} \theta \cos ^{2} \theta\right)^{-1}
\end{aligned}
$$

t In the isotropic case, the previous expression reduces to

$$
\xi=-E
$$

where $E$ is the Young modulus.

Subsequently, indicating by subscript $t$ or $c$ for tensile or compressive uniaxial stress and by subscript $x$ or $y$ its direction, the expressions of the critical hardening moduli are given by

$$
\begin{aligned}
& H_{t, x}(\theta)=\xi(\theta) \sin ^{4} \theta \\
& H_{t, y}(\theta)=\xi(\theta) \cos ^{4} \theta \\
& H_{c, x}(\theta)=\xi(\theta)\left(\frac{\beta \mu}{2} \cos ^{2} \theta+\frac{1}{\mu} \sin ^{2} \theta\right)^{2} \\
& H_{c, y}(\theta)=\xi(\theta)\left(\mu \cos ^{2} \theta+\frac{\beta}{2 \mu} \sin ^{2} \theta\right)^{2}
\end{aligned}
$$

where $\mu=\sqrt{f_{c x} / f_{c y}}$.

All the critical hardening moduli have the maximum value equal to zero, so localization starts at the onset of plastic flow for tension (since, under tension, abrupt softening occurs) and at the peak of the compressive yield stress for compression. In the case of uniaxial tension (Figures 3 4), normal $\boldsymbol{n}$ is aligned with the stress, in fact $\theta=\phi$. In the case of uniaxial compression (Figures 5, 6), for the chosen set of mechanical parameters, the expressions of the critical hardening moduli exhibit two peaks corresponding to two different possible cracks that are symmetric with respect to the compressive stress axis.

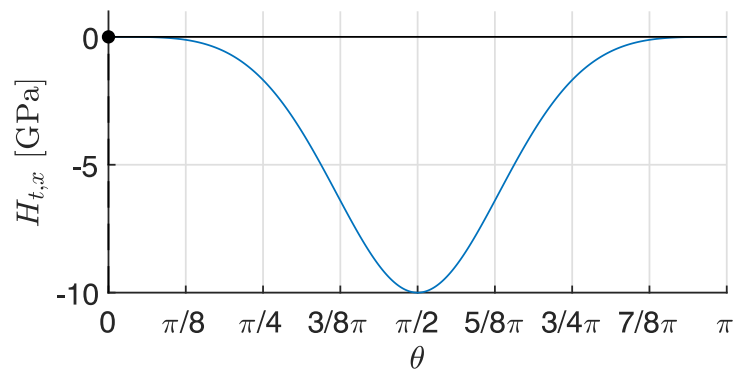

Figure 3. $H(\theta)$ for uniaxial tension in $x$-direction

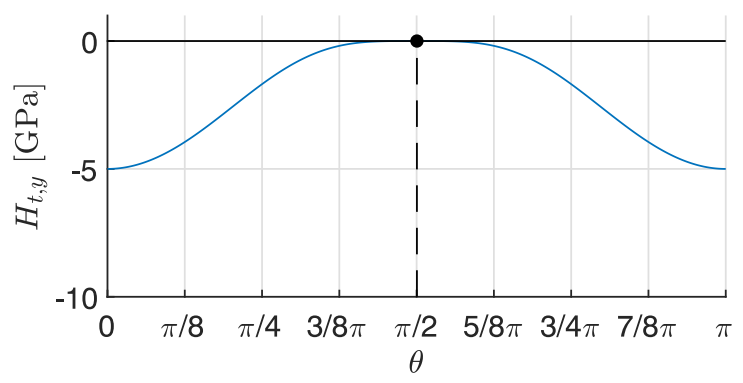

Figure 4. $H(\theta)$ for uniaxial tension in $y$-direction

The localization analysis is then extended to the generic case of uniaxial tension or compression inclined by an angle $\phi$ with respect to the $x$-axis, with $\phi \in$ $[0, \pi / 2]$. The mechanical parameters used are the same chosen by the author of the model for elementary tests under uniaxial stress [1,2] and are reported in Table 1. 


\begin{tabular}{ccccccccc}
\hline \multicolumn{3}{c}{ Elastic parameters } & \multicolumn{5}{c}{ Tensile parameters } \\
\hline$E_{x x}$ & $E_{y y}$ & $\nu_{x y}$ & $G_{x y}$ & $f_{t, x}$ & $f_{t, y}$ & $G_{t, x}$ & $G_{t, y}$ & $\alpha$ \\
{$[\mathrm{GPa}]$} & {$[\mathrm{GPa}]$} & {$[-]$} & {$[\mathrm{GPa}]$} & {$[\mathrm{MPa}]$} & {$[\mathrm{MPa}]$} & {$[\mathrm{N} / \mathrm{m}]$} & {$[\mathrm{N} / \mathrm{m}]$} & {$[-]$} \\
10.0 & 5.0 & 0.20 & 3.0 & 1.0 & 0.5 & 20 & 10 & 1.0 \\
\hline
\end{tabular}

\begin{tabular}{ccccccc}
\hline \multicolumn{8}{c}{ Compressive parameters } \\
\hline$f_{c, x}$ & $f_{c, y}$ & $G_{c, x}$ & $G_{c, y}$ & $\beta$ & $\gamma$ & $\kappa_{p}$ \\
{$[\mathrm{MPa}]$} & {$[\mathrm{MPa}]$} & {$[\mathrm{N} / \mathrm{m}]$} & {$[\mathrm{N} / \mathrm{m}]$} & {$[-]$} & {$[-]$} & {$[-]$} \\
10.0 & 5.0 & 5000 & 2500 & -1.0 & 3.0 & $5 \cdot 10^{-4}$ \\
\hline
\end{tabular}

TABLE 1. Mechanical parameters considered for localization analysis

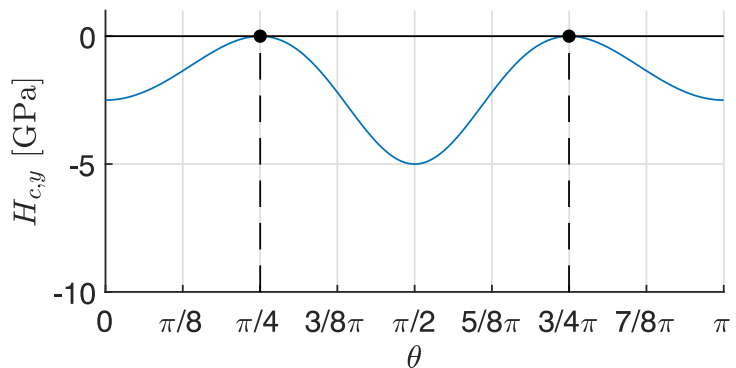

Figure 5. $H(\theta)$ for uniaxial compression in $x$-direction

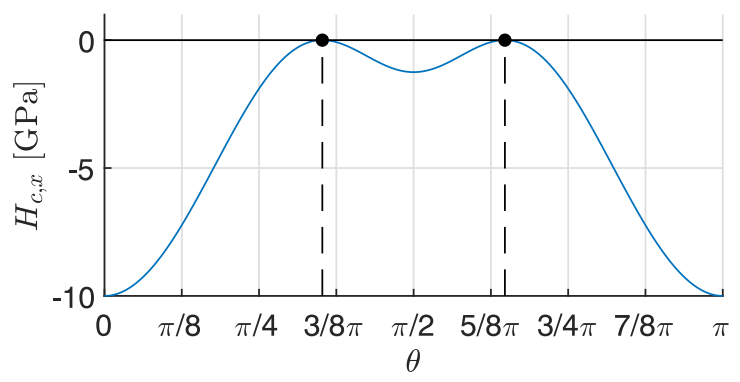

Figure 6. $H(\theta)$ for uniaxial compression in $y$-direction

The critical hardening moduli, except for the particular case of uniaxial tension along the material axes, always exhibit two maxima that correspond to the formation of two potential weak discontinuity surfaces. The solid and dashed lines represented in Figures 7 to 12 correspond to the aforementioned two solutions.

The dependence of the localization angles on the uniaxial stress angle $\phi$ for tension is represented in Figure 7, while the difference between the localization angles and the tension angle is shown in Figure 8 The variation of the inner product $\boldsymbol{n} \cdot \boldsymbol{m}$ between the normal to crack surface and the polarization vector is represented in Figure 9, which shows that for $\phi=\{0, \pi / 2\}$ its value is equal to 1 (tensile splitting), whereas for intermediate values of $\phi$ the inner product is lower, which corresponds to a misalignment between $\boldsymbol{n}$ and $\boldsymbol{m}$. It is worth noting that the two graphs are coincident since the polarization vector $\boldsymbol{m}_{1}$ corresponding to the normal $\boldsymbol{n}_{1}$ coincides with the other normal $\boldsymbol{n}_{2}$ and vice versa.

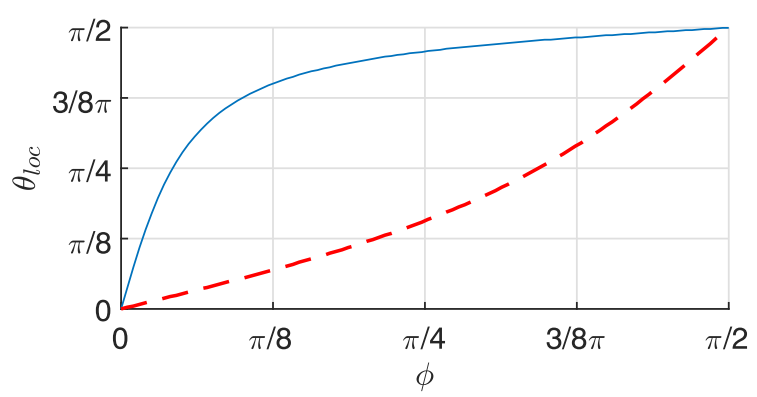

Figure 7. Dependence of $\theta_{l o c}$ on $\phi$ for uniaxial tension

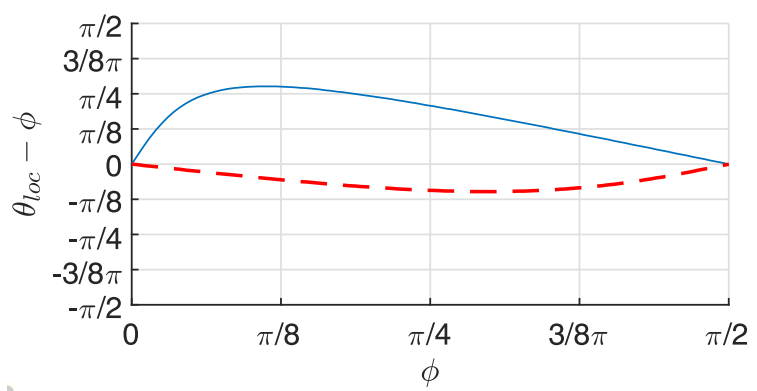

Figure 8. Difference between $\theta_{l o c}$ and $\phi$ as a function of $\phi$ for uniaxial tension

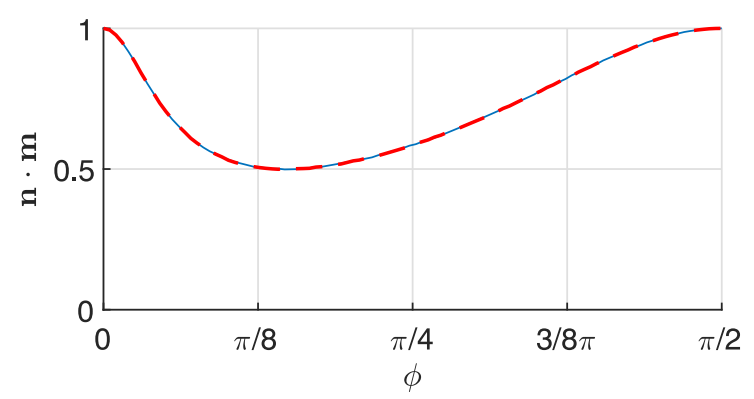

Figure 9. Dependence of $\boldsymbol{n} \cdot \boldsymbol{m}$ on $\phi$ for uniaxial tension

In the case of uniaxial compression, the dependence of the localization angles on the stress angle is reported in Figure 10. From Figure 11 one can see that the differences between the localization angles and the stress angle are almost constant and close to the values $\pi / 4$ and $3 \pi / 4$. Figure 12 shows that the graphs of 


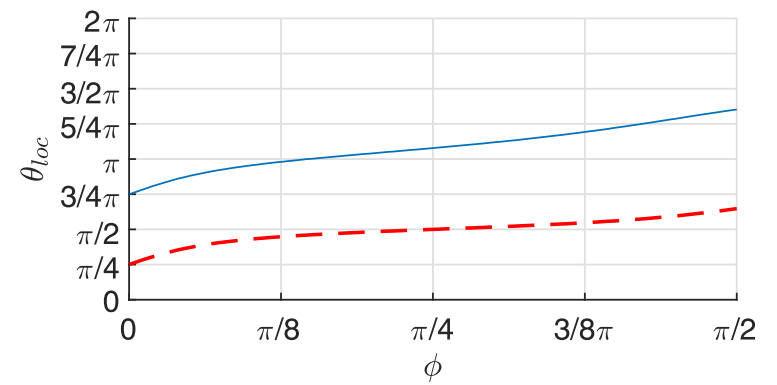

Figure 10. Dependence of $\theta_{l o c}$ on $\phi$ for uniaxial compression

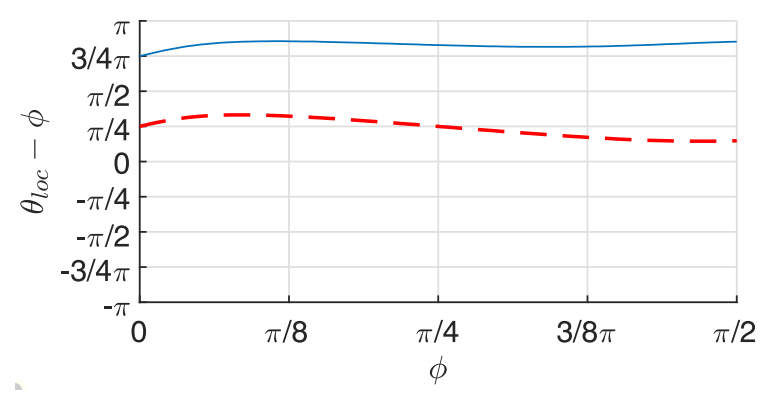

Figure 11. Difference between $\theta_{l o c}$ and $\phi$ as a function of $\phi$ under uniaxial compression

the inner products $\boldsymbol{n} \cdot \boldsymbol{m}$ corresponding to the two crack surfaces coincide as for the tensile behavior and that the polarization vector is orthogonal to the corresponding normal (shear slip) for $\phi=0$, whereas for different values of $\phi$ the inner product increases up to its maximum value for $\phi=\pi / 2$.

\section{Conclunsions}

Localization analysis under uniaxial tensile and compressive stress has been carried out.

Analytical results in the particular cases of tension or compression aligned with the material principal axes have been obtained and it has been shown that, for tensile stress, the weak discontinuity surface will form in the direction orthogonal to the stress direction while, in the case of compression, two different discontinuity surfaces, symmetric with respect to the stress direction, could develop.

The analysis has been extended by varying the angle that the stress forms with the material axes. It has been shown that for both tension and compression two localization angles can be found and some observations related to the dependence of the localization angles and the polarization vectors on the stress angle have been presented.

For all the cases described here, the localization condition is for the first time satisfied at the peak of the stress-strain diagram, i.e., the critical plastic modulus associated with the most favorable direction of a potential discontinuity is zero. For this reason, the results are not affected by the size of finite elements. The adjustment of the softening laws dependent on

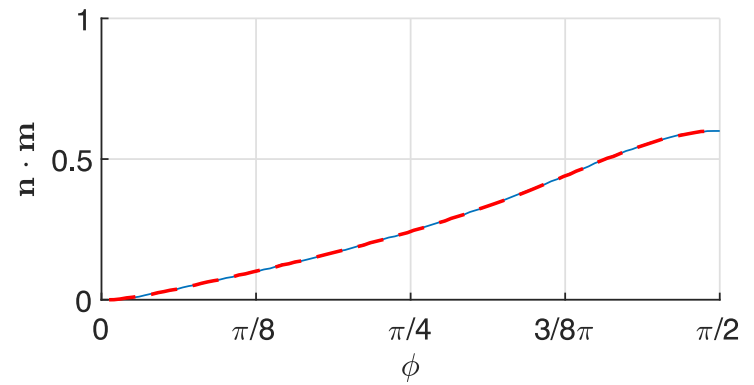

Figure 12. Dependence of $\boldsymbol{n} \cdot \boldsymbol{m}$ on $\phi$ for uniaxial compression

the element size according to 77 or $(22)$ affects only the post-localization stage of response.

\section{LIST OF SYMBOLS}

$\alpha$ Parameter that controls the shear stress contribution to tensile failure

$\beta$ Parameter that couples the normal stresses in the compressive failure surface

$\gamma$ Parameter that controls the shear stress contribution to compressive failure

$l_{e}$ Crack bandwidth

$H \quad$ Hardening modulus

$H_{\text {crit }}$ Critical hardening modulus

$\kappa_{p}$ Value of the compressive hardening parameter at peak

$f_{i, \sigma}$ Gradient of the $i$-th yield function with respect to the stress tensor

$g_{i, \sigma}$ Gradient of the $i$-th plastic potential with respect to the stress tensor

$n$ Normal to the discontinuity surface

$\boldsymbol{m}$ Polarization vector

$\phi \quad$ Uniaxial stress angle with respect to the $x$-axis

$\theta$ Angle that the generic normal to the discontinuity surface forms with the $x$-axis

$\theta_{\text {loc }}$ Localization angle

\section{ACKNOWLEDGEMENTS}

This research has been financially supported by the European Regional Development Fund through the Center of Advanced Applied Sciences at the Czech Technical University in Prague (project No. CZ.02.1.01/0.0/0.0/16_019/0000778).

\section{REFERENCES}

[1] P. Lourenço. An orthotropic continuum model for the analysis of masonry structures. Tech. rep., University of Delft, Delft, Holland, 1995.

[2] P. Lourenço. An anisotropic macro-model for masonry plates and shells: implementation and validation. Ph.D. thesis, Delft University of Technology, Faculty of Civil Engineering, 1997.

[3] M. Jirásek, M. Bauer. Numerical aspects of the crack band approach. Computers and Structures 2012. DOI:10.1016/j.compstruc.2012.06.006. 
[4] J. Hadamard. Leçons sur la propagation des ondes. Librairie Scientifique A. Hermann et Fils, Paris, 1903.

[5] R. Hill. A general theory of uniqueness and stability in elastic-plastic solids. Journal of the Mechanics and Physics of Solids 6(3):236-249, 1958.

[6] N. S. Ottosen, K. Runesson. Properties of discontinuous bifurcation solutions in elasto-plasticity. International Journal of Solids and Structures 27(4):401-421, 1991.
[7] J. W. Rudnicki, J. Rice. Conditions for the localization of deformation in pressure-sensitive dilatant materials. Journal of the Mechanics and Physics of Solids 23(6):371-394, 1975.

[8] M. Jirásek. Mathematical analysis of strain localization. Revue Européenne de Génie Civil 11(7-8):977-991, 2007.

[9] M. Jirásek, M. Horák. Localization properties of damage models. In Computational modelling of concrete structures, pp. 327-336. CRC Press, 2010. 